\title{
Estabilidade de Emulsões de Água-em-Óleo na Presença de Campo Centrífugo
}

\author{
M. Lombardo ${ }^{1}$, R. M. Charin ${ }^{1}$, M. Nele ${ }^{1,2}$, F. W. Tavares ${ }^{1,2}$ \\ ${ }^{1}$ Universidade Federal do Rio de Janeiro, Programa de Engenharia Química/COPPE \\ ${ }^{2}$ Universidade Federal do Rio de Janeiro, Departamento de Engenharia Química/EQ \\ E-mail para contato: lombardo.monique@gmail.com - tavares@eq.ufrj.br
}

RESUMO: O conceito de estabilidade de emulsões está sempre ligado à persistência ou ao decaimento do sistema disperso sob determinadas circunstâncias. A fim de estudar algumas variáveis que influenciam na estabilidade, confeccionou-se doze emulsões modelo do tipo água-em-óleo. Estas foram produzidas com diferentes percentuais de surfactante e diferentes taxas de homogeneização. Quando caracterizados segundo o comportamento de suas gotas da fase dispersa e sua reologia, os sistemas mostraram-se polidispersos e compactos, além de se comportarem como fluidos nãoNewtonianos e pseudoplásticos. Posteriormente, as doze emulsões foram submetidas à análise em campo centrífugo. Pôde-se observar um decréscimo na estabilidade com a diminuição do percentual de surfactante utilizado. Além disso, quanto mais alta a taxa de homogeneização empregada, menor o decaimento do sistema.

\section{INTRODUÇÃO}

Denomina-se emulsão uma dispersão coloidal de um líquido em outro, onde estes apresentam alto grau de imiscibilidade entre si. Tal sistema é composto por uma fase apolar, geralmente composta por hidrocarbonetos - também chamada de "óleo" ou fase oleosa - e uma fase polar, geralmente composta por água - chamada de fase aquosa.

Existem dois principais tipos de emulsões: as chamadas óleo-em-água (o/w), onde a fase apolar encontra-se dispersa na fase aquosa; e as chamadas água-em-óleo (w/o), onde a fase aquosa encontra-se dispersa na fase apolar. Porém, dependendo do tensoativo e das quantidades relativas entre as fases óelo e água, podemos encontrar emulsões múltiplas do tipo água-em-óleo-em-água $\left(\mathrm{w}_{1} / \mathrm{o} / \mathrm{w}_{2}\right)$ ou óleo-em-água-em-óleo $\left(\mathrm{o}_{1} / \mathrm{w} / \mathrm{o}_{2}\right)(\mathrm{Schramm}, 2005)$. 
Além destas, Salager (2000) relata a existência das chamadas emulsões duplas, em que estas contém dois tipos diferentes de fase dispersa, podendo ser elas de mesma natureza (mas formando gotas de tamanhos diferentes) ou de natureza diferentes (independente do tamanho de suas gotas).

O conceito de estabilidade de uma emulsão está sempre ligado ou à persistência ou ao decaimento de um sistema disperso sob determinadas circunstâncias. Salager (2000) menciona que um sistema emulsionado é dito estável quando não sofre alteração em seus aspectos por mais de três anos, e instável se sofre separação de fases após alguns minutos. Ainda segundo ele, qualquer coisa entre estes extremos é necessário quantificar de alguma forma a evolução da estabilidade com o tempo.

Vários fatores influenciam na estabilidade das emulsões, incluindo viscosidade, tamanho da gota, razão volumar da fase, temperatura, $\mathrm{pH}$, envelhecimento da emulsão, tipo do emulsificante presente, diferença de densidade e agitação. (Goodwin, 2004)

A instabilidade termodinâmica de uma emulsão é regida pela variação do tamanho das partículas dispersas, ou seja, à medida que a fase dispersa tenta se aglomerar, esta tende a se separar como uma segunda fase. Os surfactantes (emulsificantes) são adicionados ao meio de forma a retardar a segregação das fases. (Goodwin, 2004)

Métodos tradicionais de quebra incluem centrifugação, tratamento térmico, tratamento elétrico e adição de agentes químicos, contendo sabões, ácidos graxos e alcoóis de cadeia longa. (Goodwin, 2004)

A centrifugação é a técnica mais comumente aplicada para acelerar a separação de fases e desestabilizar uma emulsão. As forças tangenciais atuantes nas gotas de fase dispersa podem ser 100 vezes maiores que a força gravitacional normal. Intuitivamente, podemos pensar que quanto maior a força aplicada sobre as gotas de água mais efetivas as colisões que ocorrem entre elas e, por tanto, maior a taxa de coalescência (Krebs $e t$ al., 2012).

Os estágios de desemulsificação de emulsões w/o numa centrífuga podem ser vistos com detalhes na Figura 1. Após a preparação, a distribuição de gotas de água na emulsão é homogênea. Com a centrifugação, as gotas irão sedimentar, formando uma camada densa no fundo do recipiente. Como resultado da coalescência, uma fase de água decantada é formada, e o tamanho de gotas médio da emulsão na camada superior sofre aumento (Krebs et al., 2012).

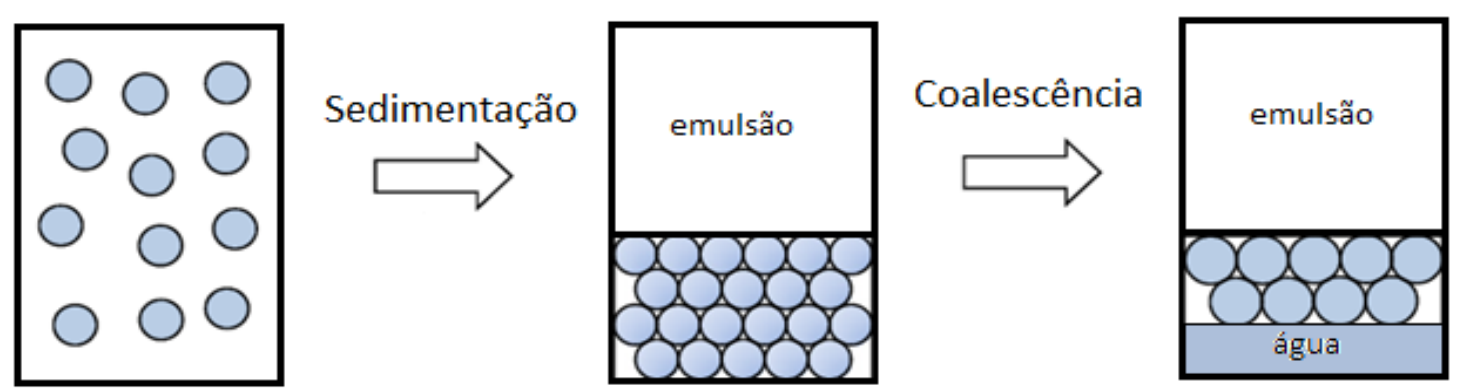

Figura 1 - Esquema de desemulsificação no campo centrífugo. O tamanho das gotas é meramente ilustrativo. 
Assim sendo, o objetivo do presente trabalho é desenvolver, caracterizar e estudar a separação de emulsões modelo do tipo água-em-óleo. As emulsões serão caracterizadas pela sua fase dispersa e sua reologia, além de serem submetidas ao estudo de estabilidade em campo centrífugo.

\section{METODOLOGIA EXPERIMENTAL}

\section{1 - Preparação das Emulsões Modelo}

As doze emulsões modelo produzidas possuem óleo de vaselina como fase contínua e água como fase dispersa. Foi verificado experimentalmente - por testes preliminares - que a mistura dos surfactantes Span 80 (preferencialmente solúvel no óleo) e Tween 80 (preferencialmente solúvel em água) é bastante eficiente. Tal mistura foi feita 3:1 em mols entre os surfactantes, e de forma que o total de surfactante presente na emulsão final variasse em $3 \%, 2 \%, 1 \%$ e $0,5 \%(\mathrm{p} / \mathrm{p})$.

Foram então preparadas doze emulsões com teor de água de $20 \%$ (p/p). Primeiramente, diluiu-se a massa correspondente de Span 80 na fase óleo a temperatura ambiente, e a massa de Tween 80 em água à quente, de forma a atingir os percentuais totais de surfactante acima mencionados. Adicionou-se, então, a fase aquosa à fase óleo e cisalhou-se no Ultra-Turrax - utilizando-se a haste modelo S25N-25F - por aproximadamente 2 minutos para homogeneizar. Variou-se também a taxa de mistura em 20.000, 15.000 e $10.000 \mathrm{rpm}$.

\section{2 - Caracterização das Emulsões Modelo}

Para observar o tamanho e o formato das gotas de fase dispersa, colocou-se uma gota de emulsão pura entre duas lamínulas previamente limpas com isopropanol. Tal sistema foi posto no microscópio Axiovert 40 MAT Inverted Microscope, da Carl Zeiss Light Microscopy, para ser observado utilizando-se a técnica de microscopia de contraste interferencial, com o auxílio do filtro DIC (Differential Interference Contrast).

As medidas de reologia das emulsões produzidas foram feitas utilizando-se um reômetro AR-G2, da TA Instruments, acoplado a um banho térmico à temperatura constante de $25^{\circ} \mathrm{C}$ e a um computador com software de comando.

Para as análises, foi utilizado a geometria placa-cone com um gap de $53 \mu \mathrm{m} \mathrm{e}$ ângulo interno $\alpha=2^{\circ}$. Aplicou-se sobre as amostras uma taxa de cisalhamento que variou de $0-200 \mathrm{~s}^{-1}$ por 5 minutos. Foram feitas duplicatas. Os resultados foram analisados mostrando o comportamento reológico das amostras para os diferentes percentuais totais de surfactante e diferentes taxas de mistura no Turrax.

Além disso, foi utilizado o mesmo procedimento experimental para traçar o comportamento reológico da fase óleo original (óleo mineral) e compará-lo com o perfil das emulsões produzidas. 


\section{3 - Estudo de Estabilidade em Campo Centrífugo}

Cada uma das doze emulsões foram submetidas ao campo centrífugo em triplicata, a uma temperatura de $60^{\circ} \mathrm{C}$, uma rotação de $2.000 \mathrm{rpm}$ e um tempo total de análise de 2 horas. Foi utilizada para conduzir os experimentos a centrífuga com aquecimento modelo NT870, da Nova Técnica Equipamentos para Laboratório. Para determinar o percentual de água decantada em cada centrifugação, utilizou-se a seguinte metodologia:

i. Pesou-se por diferença a massa de emulsão contida em cada tubo de centrífuga.

ii. Assumindo a hipótese de mistura perfeita, afirmou-se que $20 \%$ da massa de emulsão em cada tubo é composta por água.

iii. Após colocar os tubos na centrífuga nas condições descritas acima, anotou-se o volume de água decantada no fim dos seguintes tempos: 30 minutos, 1 hora, 1 hora e 15 minutos, 1 hora e 30 minutos, 1 hora e 45 minutos e 2 horas.

iv. Assumindo a hipótese que a densidade da água separada é igual a densidade da água pura, transformou-se o volume de água separada em massa para calcular o percentual de água decantada.

v. Confeccionaram-se gráficos para mostrar o percentual de água decantada vs. tempo, para os diferentes percentuais totais de surfactante e diferentes taxas de mistura no Turrax.

\section{RESULTADOS E DISCUSSÕES}

As doze emulsões produzidas, segundo o procedimento anteriormente explícito, são opacas, de cor branca, têm aspecto oleoso, não são voláteis e não possuem odor característico. Todas elas são estáveis à temperatura e pressão ambiente, sem apresentar separação de fases visível por vários meses.

\section{1 - Caracterização das Emulsões Modelo}

Ao observar a amostra de emulsão no microscópio, foi visto que trata-se de um sistema polidisperso e compacto, ou seja, as gotas formadas possuem uma variada distribuição de tamanhos e, além disso, encontram-se bem próximas umas das outras. Tais gotas são pequenas, menores que $2 \mu \mathrm{m}$.

Para determinar a DTG por microscopia, é necessário que a amostra de emulsão seja diluída, de forma que se possa obter imagens mais definidas das interfaces das gotas. O ideal seria diluí-la em óleo mineral puro. Porém, devido a insolubilidade em sua fase óleo original, testou-se a solubilidade em vários solventes orgânicos de diversas polaridades, seguindo a seguinte série eluotrópica (série de Trappe):

$$
\begin{gathered}
\text { tetracloreto de carbono }<\text { tolueno }<\text { clorofórmio }<\text { éter etílico } \\
<\text { acetato de etila }<\text { acetona }<\text { etanol }<\text { metanol }
\end{gathered}
$$


Foi constatado que a emulsão não apresentava solubilidade em nenhum dos solventes testados. Para tanto, é possível concluir que as emulsões possuem gotas que interagem fortemente entre si, de forma que esta interação não permite que os solventes penetrem na camada de fase contínua entre as gotas, afastando-as.

Assim, apesar de ter sido útil para a caracterização do comportamento das emulsões, devido a esta impossíbilidade de diluição da amostra, torna-se inviável a medição mais precisa de distribuição de tamanho de gotas através da técnica de microscopia ótica.

Para as análises de reologia, primeiramente, foi estudado o comportamento da fase óleo original, ou seja, do óleo mineral puro. Segundo medido, o óleo mineral utilizado é um líquido Newtoniano, com viscosidade média $\eta=0,1429$ Pa.s. Ao compararmos sua viscosidade com a da água pura à $25^{\circ} \mathrm{C}(\eta=0,891 \mathrm{mPa} . \mathrm{s})$, podemos perceber o quanto $\mathrm{o}$ óleo é mais viscoso que a água.

Então, cada uma das doze emulsões foram analizadas reologicamente em duplicata. Através dessas análises foi possível observar que todas elas são fluidos nãoNewtonianos e pseudoplásticos, com viscosidades dinâmicas maiores que a viscosidade do óleo mineral puro.

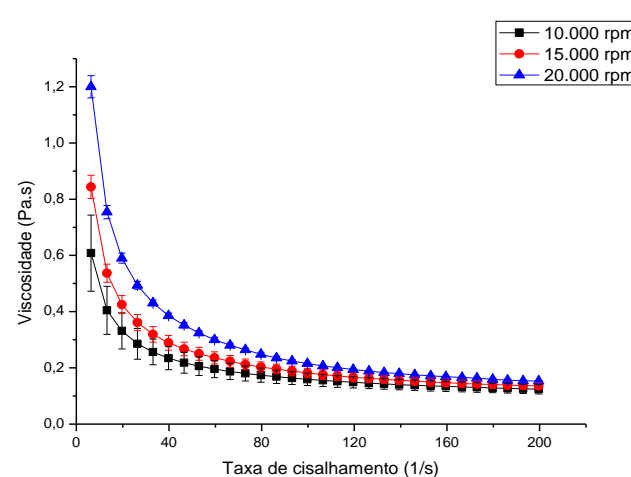

(a)

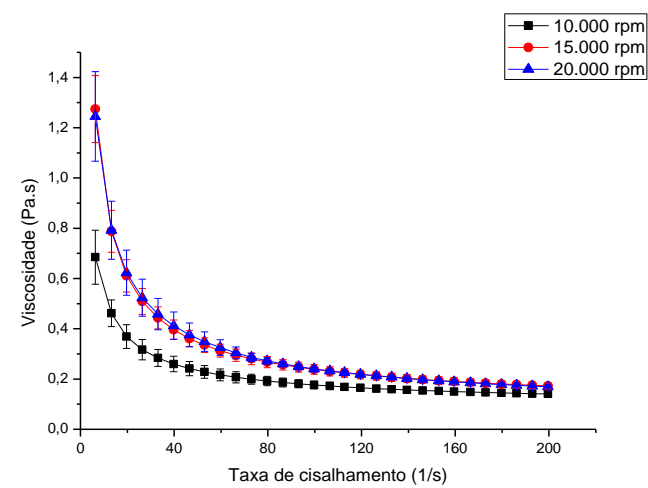

(c)

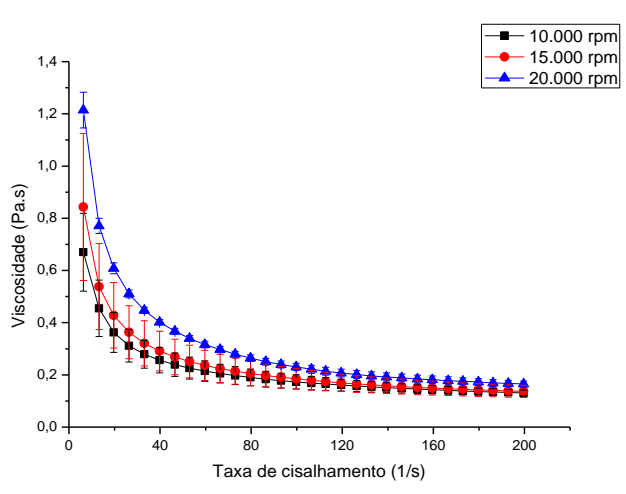

(b)

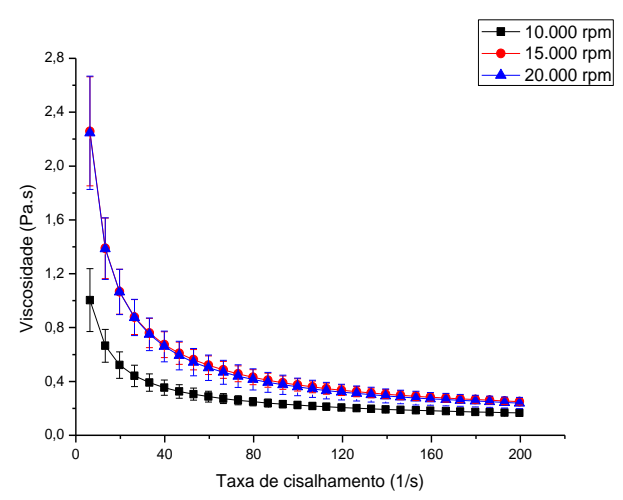

(d)

Figura 2 - Curvas de viscosidade das emulsões contendo (a) 3\%, (b) $2 \%$, (c) $1 \%$ e (d) $0,5 \%$ de surfactante. 
Podemos encontrar na literatura relatos de Salager (2000) mencionando que, para o caso em que a fase óleo é composta de óleos parafínicos mais viscosos que a água, como esperado da relação entre a viscosidade da emulsão e a viscosidade de sua fase contínua, emulsões de w/o são bem mais viscosas que suas emulsões o/w homólogas. Tal observação leva a concluir que as doze emulsões formadas são realmente emulsões de água-em-óleo, como desejado.

\section{2 - Estudo de Estabilidade em Campo Centrífugo}

Segundo o procedimento explicitado anteriormente, as análises de estabilidade em campo centrífugo foram feitas à temperatura de $60^{\circ} \mathrm{C}$ e com rotação de $2.000 \mathrm{rpm}$. Cada uma das doze emulsões foi analisadas em triplicata.

Como resultado, foram construídos gráficos de percentual de água decantada vs. tempo de experimento, para as diferentes taxas de mistura do turrax (Figura 3). Através desses gráficos, foi possível observar que quanto menor o percentual de surfactante utilizado para a produção da emulsão, maior o percentual de água decantada, para qualquer taxa de mistura utilizada.

Uma possível explicação seria que quanto menor a concentração de surfactante utilizada para produzir a emulsão, menor também a concentração de surfactante nas interfaces das gotas de fase dispersa e, portanto, estas são mais suscetíveis à coalescência e à sedimentação, diminuindo a estabilidade e aumentando o percentual de água decantada. Logo, numa escala crescente de estabilidade, temos:

\section{$0,5 \%$ surfactante $<1 \%$ surfactante $<2 \%$ surfactante $<3 \%$ surfactante}
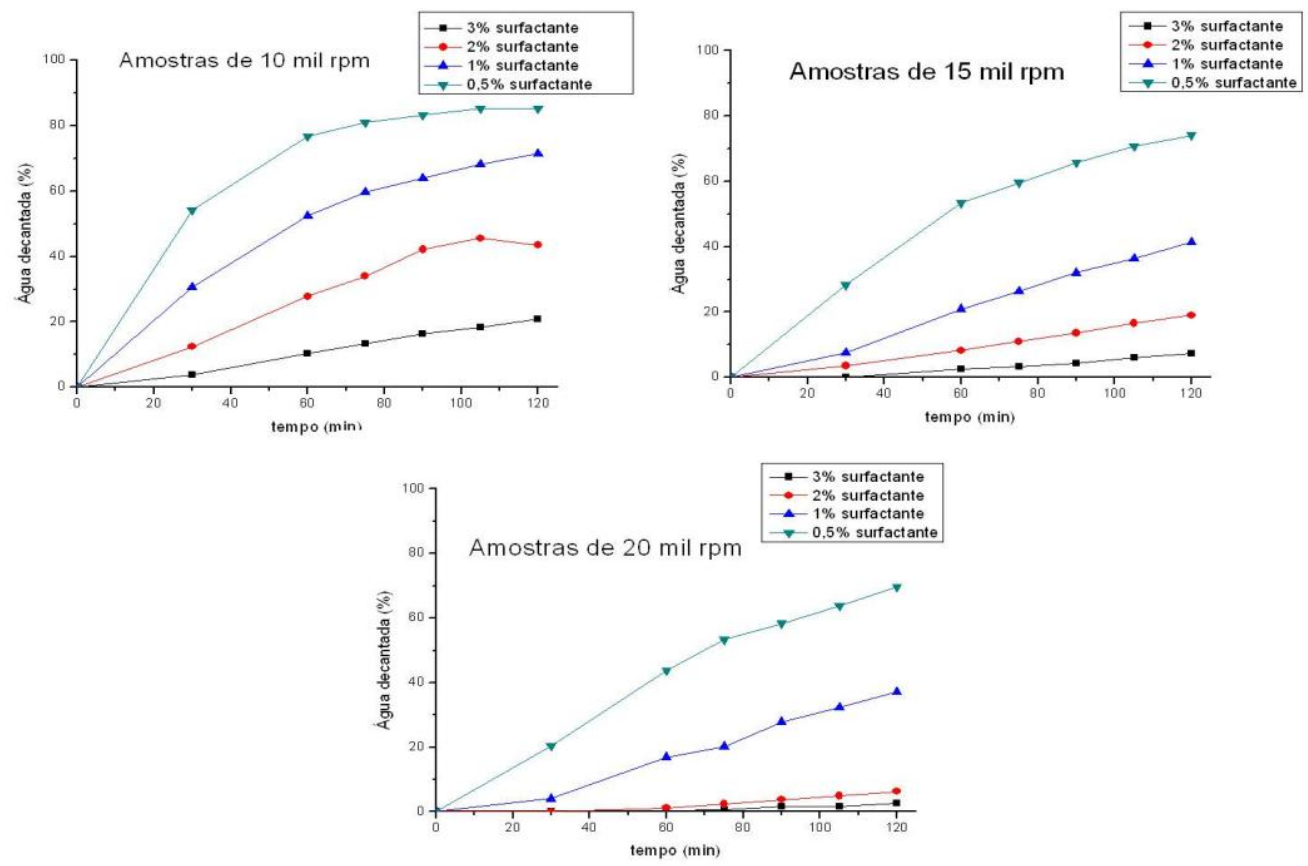

Figura 3 - Gráficos de percentual de água decantada vs. tempo para as diferentes taxas de mistura. 
Além disso, com os mesmos dados obtidos, foram feitos gráficos comparativos de percentual de água decantada vs. tempo de experimento para os diferentes percentuais de surfactante. Através desses gráficos, é possível observar que quanto maior a taxa de mistura utilizada para a produção da emulsão, menor o percentual de água decantada, independente do percentual de surfactante utilizado.

Uma possível explicação seria que quanto maior a taxa de mistura utilizada para produzir a emulsão, menor o tamanho das gotas de fase dispersa e, portanto, maior a estabilidade, o que diminui o percentual de água decantada. Logo, numa escala crescente de estabilidade, temos:

$$
10 \text { mil rpm }<15 \text { mil rpm }<20 \text { mil rpm }
$$
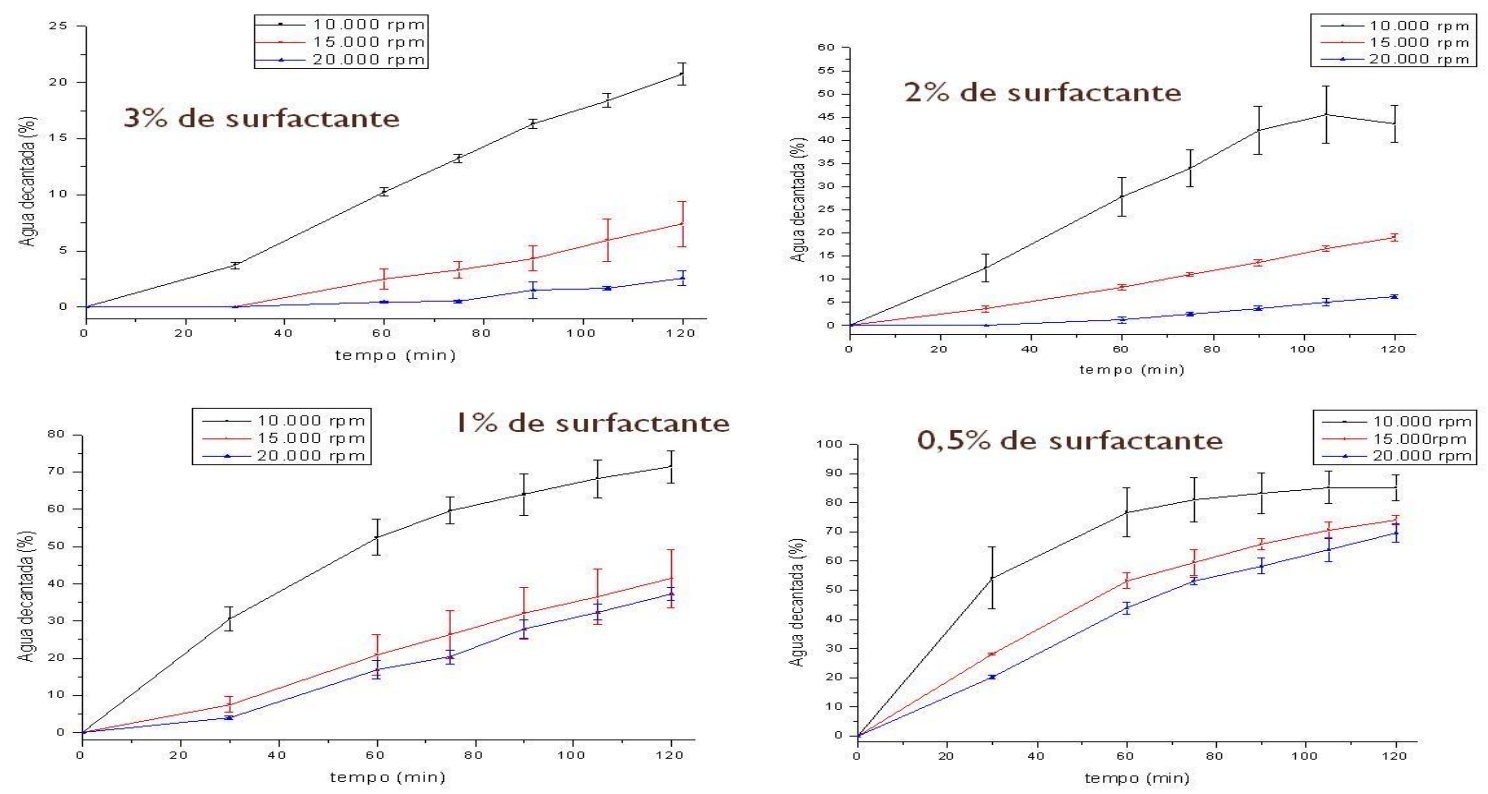

Figura 4 - Gráficos de percentual de água decantada vs. tempo para os diferentes percentuais de surfactante.

\section{CONCLUSÕES}

Foi objetivo deste trabalho desenvolver, caracterizar e avaliar a estabilidade de emulsões modelos do tipo água-em-óleo. Foram desenvolvidos doze sistemas emulsionados diferentes, contendo óleo mineral como fase contínua, água deionizada como fase dispersa, e uma mistura 3:1 em mols de Span 80 e Tween 80 como estabilizante. Nesses sistemas, variou-se o percentual de surfactante - em 3\%, 2\%, $1 \%$ e $0,5 \%(\mathrm{p} / \mathrm{p})$ - e a taxa de mistura das fases - em 20000, 15000 e $10000 \mathrm{rpm}$. 
A caracterização das gotas de fase dispersa foi feita utilizando-se a microscopia de contraste interferencial. Ao observar uma gota de emulsão pura, foi visto que tratavamse de sistemas polidispersos e compactos, com gotas menores que $2 \mu \mathrm{m}$. Porém, devido à insolubilidade das emulsões, tanto em sua fase óleo original quanto nos solventes orgânicos testados, tornou-se inviável aferir a distribuição de tamanhos de gota.

As medidas de comportamento reológico das doze emulsões mostraram que todas são não-Newtonianas e pseudoplásticas. A partir destas, foi possível extrair que todas as emulsões possuíam viscosidades aparentes maiores que a fase óleo original, o que confirmou que elas são mesmo do tipo w/o.

As análises de estabilidade em campo centrífugo permitiram avaliar que tanto o percentual de surfactante quanto a taxa de mistura afetam a estabilidade da emulsão. Pôde-se concluir que um decréscimo no percentual de surfactante diminui a estabilidade da emulsão. Além disso, quanto maior a taxa de mistura, mais estáveis são as emulsões.

\section{REFERÊNCIAS BIBLIOGRÁFICAS}

BECHER, P. Emulsions theory and practice. $3^{\text {rd }}$ edition, New York, Oxford University Press Inc., 1957.

GOODWIN, J.W. Colloids and Interfaces with Surfactants and Polymers - An Introduction. England, John Wiley and Sons LTD, 2004.

KREBS, T.; SCHROËN, C.G.P.H.; BOOM, R.M. "Separation kinetics of an oil-in-water emulsion under enhanced gravity”. Chemical Engineering Science, v. 71, pp. 118-125, 2012.

SALAGER, J.L. "Emulsion Properties and Related Know-how to Attain Them". In: MARTIMESTRES, G.; NIELLOUD, F. Pharmaceutical emulsions and suspensions, cap. 2 e 3, New York, Marcel Dekker Inc., 2000.

SCHRAMM, L.L. Emulsions, Foams and Suspensions: Fundamentals and Applications. Germany, Wiley, VCH, 2005. 